\title{
Pedestal free pulse compression of chirped optical solitons
}

\author{
K. Senthilnathan, ${ }^{1}$ K. Nakkeeran, ${ }^{2}$ Qian $\mathrm{Li}^{3},{ }^{3}$ and P. K. A. Wai ${ }^{3}$ \\ ${ }^{1}$ Photonics Division, Department of Physics, \\ School of Advanced Sciences, VIT University, \\ Vellore - 632 014, Tamil Nadu, India \\ ${ }^{2}$ School of Engineering, Fraser Noble Building, King's College, \\ University of Aberdeen, Aberdeen AB24 3UE, Scotland, UK \\ ${ }^{3}$ Photonics Research Center and Department of Electronic and Information Engineering, \\ The Hong Kong Polytechnic University, Hung Hom, Kowloon, Hong Kong
}

(Dated: May 17, 2012)

We consider the evolution of nonlinear optical pulses in inhomogeneous optical media wherein the pulse propagation is governed by the nonlinear Schrödinger equation with varying dispersion. We adopt the Painlevé analysis in order to obtain the condition for the soliton pulse propagation. We find that there exist two dispersion profiles satisfying this criterion, namely, the constant dispersion and exponentially dispersion decreasing profiles. In the exponentially varying dispersion media, we explain the existence and the formation of chirped optical soliton through the equation for the chirp parameter that results from variational analysis. For further elucidation, we provide the phase-plane diagram in terms of the normalized chirp and intensity (peak power), which explains the physical mechanism of linearly chirped soliton pulse compression in the exponentially dispersion decreasing media. In addition, we discuss the generation of exact chirped higher order solitons using the Bäcklund transformation method. As a special case, we find an oscillatory two-soliton breather pulse, which, at decreasing intervals, evolves into a familiar hyperbolic secant shape whose amplitude is twice that of a fundamental soliton. We highlight on the point that the crux of this work in realizing a compact pulse compressor could possibly result in a myriad of applications in modern optical fiber communications systems.

PACS numbers: 


\section{INTRODUCTION}

In optical fibers, a dynamical balance occurs between a linear effect called anomalous group velocity dispersion (AGVD) and a nonlinear effect called self-phase modulation (SPM). While AGVD induces a negative chirp, SPM brings in a positive chirp. Hence, the resulting pulse exhibits zero chirp in its evolution, and the same is called a soliton [1-4]. Soliton pulse propagation in optical fibers is governed by the well-known nonlinear Schrödinger equation (NLSE) [1-4]. Although an ideal fundamental soliton is very robust, the pulse propagation in real optical fiber systems does face many limitations, especially, on the design of the communication system. Of all the issues, fiber loss turns to be the most detrimental in communications. Nonetheless, this problem is being addressed very well, in recent times, by using optical amplifiers [2]. An alternate approach for this loss compensation stems from using fibers with exponentially decreasing dispersion profile [2]. In modern technology, this desired profile can easily be fabricated by tapering the core of the optical fiber. In 1995, for the first time, exponentially decreasing dispersion fibers over lengths $38 \mathrm{~km}$ and $40 \mathrm{~km}$ were fabricated for compensating the fiber loss [5, 6]. In this paper, we demonstrate the existence of bizarrely chirped solitons in optical fibers with exponential decreasing dispersion profile. Contrary to the solitons in fibers with constant dispersion profiles, these solitons are the chirped ones. We further investigate these chirped solitons and their applications for achieving effective pulse compression in exponentially decreasing dispersive media.

Soliton pulse compression and adiabatic pulse compression techniques are most commonly used ones for the compression process. In soliton pulse compression, the compressed pulses suffer from significant pedestal generation, leading to nonlinear interactions between neighboring solitons [7]. On the other hand, adiabatic pulse compression provides highly compresssed pulses with minimal pedestals. Nevertheless, it is difficult to maintain the adiabatic condition throughout the compression process [7]. Thus, soliton effect compression and adiabatic compression do not meet all the desired expectations. It has been demonstrated that going into higher order solitons results in higher compression factor. However, the compressed pulses do possess large pedestals owing to the absence of linear chirp. Therefore, it has become the need of the hour to develop a compression technique which should be capable of generating high quality pulses with large compression factor. 
Instead of chirp-free solitons, more recently, attention has been riveted on the generation of chirped solitons. Chirped solitary waves can be compressed more efficiently if the dispersion decreases approximately exponentially [8]. Recently, self-similar analysis has been employed to obtain the linearly chirped solitary wave pulses which, indeed, facilitate efficient compression or amplification [9]. Very recently, using the self-similar analysis, the pedestal-free Bragg soliton pulse compression has been examined [10]. In addition, FBGs with the stepwise approximation of the exponentially decreasing dispersion have also been proposed to generate the nearly transform-limited pulses [11]. In this paper, we describe the existence and the formation of chirped optical solitons in exponentially decreasing dispersive media. The paper is organized as follows. In Section II, we demonstrate that exponentially decreasing dispersion profile of the governing media is a condition for soliton pulse propagation using Painlevé analysis. It would also mean that the NLSE with variable dispersion coefficient is integrable only when the dispersion varies exponentially. We derive the exact chirped one-soliton solution using the Bäcklund transformation (BT) method in Section III. By variational analysis, we arrive at the physical explanation for the formation of chirped optical solitons in Section IV. In Section V, we show the implementation of the chirped optical soliton in terms of an effective chirp-free and pedestal-free pulse compression technique. Next, we discuss the existence of chirped higher order solitons in Section VI. We address the evolution and interaction of the chirped higher order solitons in Section VII. In addition, we delineate a special chirped soliton pulse, known as chirped soliton breather, whose amplitude is twice that of the fundamental soliton at decreasing intervals. Finally, we conclude in Section VIII.

\section{THEORETICAL MODEL}

We consider pulse propagation in a nonlinear medium wherein the dispersion varies along the propagation direction. Pulse propagation in such a medium is governed by the variable coefficient NLSE

$$
i \psi_{z}-\frac{\beta(z)}{2} \psi_{t t}+\gamma|\psi|^{2} \psi=0
$$

where $\psi$ is the slowly varying envelope of the axial electrical field, $\beta(z)$ and $\gamma$ represent the group-velocity dispersion parameter which varies along the propagation direction and the 
SPM parameter, respectively. The variables $z$ and $t$ represent distance and time, respectively. Although these definitions of the variables and parameters are for the context of envelope soliton propagation in optical fibers, they get changed for other physical systems governed by the NLSE [12]. The variable coefficient NLSE (1) also governs the dynamics of dispersionmanaged (DM) optical fiber solitons which is another important type of pulse propagation in fibers useful for high-speed data transmission [2]. Equation (1) has been extensively investigated by many researchers in different contexts [9, 13-16].

To check the possibility of soliton pulse propagation in dispersion varying media, we apply the well known Painlevé analysis to Eq. (1). The Painlevé analysis demands that the dispersion vary in an exponential manner or else remain constant for the system Eq. (1) to be completely integrable. That is, soliton pulse propagation is possible only if the dispersion varies exponentially as

$$
\beta=\beta_{0} \exp (-\sigma z)
$$

where $\beta_{0}$ and $\sigma$ are integration constants. It should be emphasized that the exponential scaling (for the dispersion profile), obtained here by Painlevé analysis, is the same as that of the one obtained through self-similar analysis [9]. As has been discussed in the introduction, 38-km and 40-km of exponentially decreasing dispersion fibers had been fabricated to compensate for the fiber loss [5, 6]. The integrable form of Eq. (1) can be written as (with $\left.\sigma=\beta_{0} \alpha_{0}\right)$

$$
i \psi_{z}-\frac{\beta_{0} \exp \left(-\beta_{0} \alpha_{0} z\right)}{2} \psi_{t t}+\gamma|\psi|^{2} \psi=0
$$

We would now like to point out a conjecture regarding the resonance values derived in the Painlevé analysis. The resonances $r=-1,0,3,4$ obtained here for the variable coefficient NLSE (1) are the same as that of the constant coefficient NLSE. Past experience has shown that such coincidence usually implies that the newly derived integrable nonlinear evolution equation can be connected to existing systems of equations. This is in fact true and there is a connection between the variable coefficient NLSE (3) and the conventional NLSE. Now, we consider the gauge transformations

$$
\psi \rightarrow \psi \exp \left(i \alpha_{0} t^{2} / 4\right) ; \quad t \rightarrow t \exp \left(-\beta_{0} \alpha_{0} z / 2\right)
$$


which map the exponentially varying dispersion NLSE (3) into the following variable coefficient NLSE:

$$
i \psi_{z}-\frac{\beta_{0}}{2} \psi_{t t}+\gamma|\psi|^{2} \psi-\frac{\beta_{0}}{8} \alpha_{0}^{2} t^{2} \psi-i \frac{\beta_{0}}{4} \alpha_{0} \psi=0
$$

The variable coefficient NLSE (5) has been analyzed for its integrability through Painlevé analysis and is found to possess a transformation relating to constant coefficient NLSE [17]. Thus the above mentioned conjecture about the resonance values of the Painlevé analysis holds good as there is a connection between the integrable dispersion varying NLSE (3) and the conventional constant coefficient NLSE. Equation (5) is also applicable in many physical contexts such as averaged DM fiber system, nonlinear compression of chirped solitary waves, quasi-soliton propagation in DM optical fiber, etc [8, 18-20]. Although the results on Painlevé analysis for the NLSE with the variable coefficients have already been reported in the literature, we emphasize over the fact that, till date, there has been no clear discussion on the existence of chirped optical solitons in the nonlinear optical media and eventually, this issue turns out to be the ultimate crux of this paper.

\section{CHIRPED OPTICAL SOLITON: BÄCKLUND TRANSFORMATION METHOD}

Now we present the essential steps for deriving the exact chirped soliton solution of the variable coefficient NLSE (5) using the Bäcklund transformation method. Before investigating the chirped soliton, it is customary to construct the Lax pair associated with Eq. (5). In order to construct the chirped soliton of Eq. (5), we start with the zero-soliton solution $Q=0$. By using this trivial solution, the explicit form of $\Gamma(0)$ is obtained as

$$
\Gamma(0)=\exp [\zeta(z, t)+i \theta(z, t)]
$$

where $\zeta(z, t)$ and $\theta(z, t)$ are given by

$$
\begin{aligned}
& \zeta(z, t)=-4 \beta_{0} \int u(z) v(z) d z+2 v(z) t \\
& \theta(z, t)=2 \beta_{0} \int\left[u^{2}(z)-v^{2}(z)\right] d z-2 u(z) t
\end{aligned}
$$


The explicit form of $\zeta$ and $\theta$ can be derived from the above two equations using the variable spectral parameter. The exact 1-soliton solution for the exponentially decreasing dispersion NLSE (3) can be derived as

$$
\psi(1)=\sqrt{\frac{-\beta_{0}}{\gamma}} \frac{e^{\frac{\chi}{2}}}{T_{0}} \operatorname{sech}\left(\frac{t e^{\chi}}{T_{0}}\right) \exp \left[\frac{i}{2}\left(\alpha_{0} t^{2}-\frac{1}{\alpha_{0} T_{0}^{2}}\right) e^{\chi}\right],
$$

where $\chi=\alpha_{0} \beta_{0} z$. For simplicity, we assume $\lambda_{0 I}=1 /\left(2 T_{0}\right)$ and $\lambda_{0 R}=0$, where $T_{0}$ is a real constant which represents the initial pulse width parameter. This soliton solution is similar to that of chirped soliton obtained by self-similar analysis reported in the literature $[8,9,16]$. From the factor $\chi$ in the 1 -soliton solution $(9)$, it is clear that the soliton pulse intensity and chirp increase exponentially and the width decreases exponentially at the same rate along the propagation direction. The conventional chirp-free soliton formation is usually explained based on the dispersion length $\left(L_{D}=T_{0}^{2} /\left|\beta_{0}\right|\right)$ and nonlinear length $\left(L_{N L}=1 /(\gamma P)\right)$, where $P\left(=\left|\beta_{0}\right| / \gamma / T_{0}\right)$ is the peak intensity of the pulse. In a similar way, to explain the chirped soliton formation, we need to define another length scale called chirp length, $L_{C}=1 /\left(\alpha_{0} \beta_{0}\right)$, to take into account the effect of chirp. Note that the dispersion and nonlinear lengths decrease exponentially while chirp length is constant during the formation of chirped solitons. In this context, the concept of chirp length emerges in a natural way as dispersion and nonlinear lengths.

\section{PHYSICAL EXPLANATION OF CHIRPED OPTICAL SOLITON: VARIATIONAL ANALYSIS}

To explain the dynamics of the chirped optical solitons we make use of the following variational analysis [21]. When we consider the hyperbolic secant ansatz

$$
\psi=x_{1} \operatorname{sech}\left(\frac{t}{x_{2}}\right) \exp \left(i x_{3} \frac{t^{2}}{2}+i x_{4}\right),
$$

in the variational analysis of the NLSE (1), the evolution equations for the pulse amplitude $\left(x_{1}\right)$, width $\left(x_{2}\right)$, chirp $\left(x_{3}\right)$ and phase $\left(x_{4}\right)$ are derived as: 


$$
\begin{aligned}
& \frac{d x_{1}}{d z}=\frac{\beta(z)}{2} x_{1} x_{3}, \\
& \frac{d x_{3}}{d z}=\beta(z)\left(x_{3}^{2}-\frac{4}{\pi^{2} x_{2}^{4}}\right)-\frac{4 \gamma x_{1}^{2}}{\pi^{2} x_{2}^{2}}, \\
& \frac{d x_{2}}{d z}=-\beta(z) x_{2} x_{3} \\
& \frac{d x_{4}}{d z}=\frac{\beta(z)}{3 x_{2}^{2}}+\frac{5 \gamma x_{1}^{2}}{6} .
\end{aligned}
$$

As we are interested in the formation of chirped soliton, we focus only on the chirp parameter to explain the relation amongst the dispersion length, nonlinear length and the chirp length. In particular, the evolution equation of the chirp can be written as

$$
\frac{d x_{3}}{d z}=\beta(z) x_{3}^{2}+\frac{4}{\pi^{2} x_{2}^{2}}\left(\frac{1}{L_{D}}-\frac{1}{L_{N L}}\right),
$$

where $L_{D}=-x_{2}^{2} / \beta(z)$ and $L_{N L}=1 /\left(\gamma x_{1}^{2}\right)$, which, respectively, represent the dispersion and nonlinear lengths. In what follows, we discuss the possibility of chirp-free solitons and chirped solitons in the optical systems which are governed by the integrable NLSE (3).

Chirped solitons :- The same integrability condition (2) for a lossless fiber can also support a chirped optical soliton. From the soliton solution (9), we can get the expressions for the pulse width and intensity as $x_{2}=x_{20} \exp (-\chi)$ and $x_{1}^{2}=x_{10}^{2} \exp (\chi)$ (where $x_{10}$ and $x_{20}$ are initial pulse amplitude and width, respectively). From the integrability condition the dispersion can decrease exponentially as $\beta(z)=\beta_{0} \exp (-\chi)$. Thus the dispersion and nonlinear lengths for the chirped soliton propagation are given by $L_{D}=-x_{20}^{2} \exp (-\chi) / \beta_{0}$ and $L_{N L}=\exp (-\chi) /\left(\gamma x_{10}^{2}\right)$, respectively. Unlike the situation in conventional optical soliton, the dispersion and nonlinear lengths decrease in the same way as the dispersion along the propagation direction in the case of chirped soliton. The dispersion length is equal to the nonlinear length at every point of the fiber. This is possible only because of the evolution of the chirp. When we substitute the expressions for the dispersion, pulse width, linear length $\left(L_{D}\right)$ and the nonlinear length $\left(L_{N L}\right)$ in the chirp evolution equation (11), we get $d x_{3} / d z=\beta_{0} \exp (-\chi) x_{3}^{2}$. If we consider the initial chirp of the soliton pulse as $\alpha_{0}$, then the chirp would evolve as $x_{3}=\alpha_{0} \exp (\chi)$. This is also obvious from the 1-soliton solution (9) derived for the dispersion varying NLSE (3). In the case of conventional optical soliton, 
the chirp produced by the dispersion is balanced by the chirp produced by the nonlinearity. In the variable coefficient NLSE (3) the dispersion parameter decreases exponentially and the nonlinear parameter remains a constant. Thus we can say that, in the absence of any chirp, the chirp produced by the linear effect is not balanced by the nonlinear effect. Hence, it looks like the nonlinear effect overwhelming the linear effect. However, the additional chirp, present initially in the pulse (the sign of this initial chirp is the same as that of the chirp produced by the anomalous dispersion fiber) grows exponentially and adds to the chirp generated by the dispersion to balance with the nonlinear chirp for the formation of chirped solitons. Thus these chirped optical solitons are formed basically because of the growth of the chirp in contrast to the zero chirp in the case of the conventional solitons. Very recently, we have observed that the chirped solitons evolve in a different manner when compared to chirp-free solitons [10].

\section{DEPLOYING CHIRPED SOLITON: PULSE COMPRESSION}

Now, we turn to discuss the deployment of chirped soliton as an effective pulse compression technique. Fig. 1 explains the phase-plane diagram in terms of normalized chirp and peak power. The dot-dashed curve represents the pre-chirping of an initial chirp-free hyperbolic secant pulse that results from the pulse parameter equations under the condition $\gamma=0$. Once the required chirp is obtained, the pulse is sent to a nonlinear medium with an exponentially decreasing dispersion profile. Hence the pulse is compressed and the normalized chirp also gets decreased in the compression process (solid curve). The residual chirp can be removed by the de-chirping process (dashed curve).

In Fig. 1, the pulse compression has been analyzed for two different values of initial chirp. In the first case, we consider the initial normalized chirp to be $C(z)=-0.4012$. During the compression, the normalized chirp decreases and eventually the compressed pulse possesses a small amount of normalized chirp $C(z)=-0.1046$. If the initial normalized chirp is relatively large, $C(z)=-1.3374$, the pulse undergoes, relatively, a higher compression as shown in Fig. 1. After the compression process, the normalized chirp gets reduced to -0.1373 . In both the cases, the compressed pulses possess a small amount of chirp. Thus, at the end, chirp-free pulses are achieved by a suitable de-chirping process (dashed curve).

As discussed in the introduction, the dispersion varying NLSE (3) governs the nonlinear 


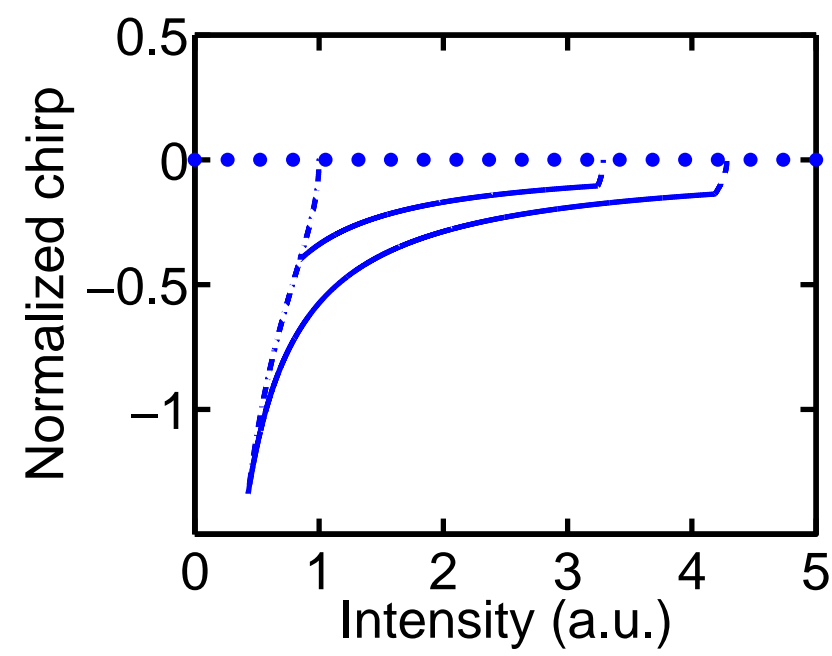

FIG. 1: Phase plane diagram for the chirped soliton pulse compression in terms of normalized chirp and intensity.

pulse propagation in dispersion decreasing fibers and also near the photonic band gap of a nonuniform FBG $[22,23]$. In the present study, FBG has been preferred as it possesses a huge amount of grating induced dispersion of six orders of magnitude larger than the conventional telecommunication fiber. The presence of large dispersion in FBG allows the study of soliton dynamics on the length scales of centimeters. Thus, one can realize very short and efficient pulse compressors with high degree of compression and pulses of good quality. In this work, we assume a nonuniform FBG of length $L=6 \mathrm{~cm}$ with the initial dispersion of $\beta(0)=-33 \mathrm{ps}^{2} \mathrm{~cm}^{-1}$. We take the effective core area of the FBG as $20 \mu \mathrm{m}^{2}$ and the nonlinear coefficient as $2.3 \times 10^{-16} \mathrm{~cm}^{2} \mathrm{~W}^{-1}$. The initial dispersion monotonically decreases to a final value at the end of the dispersion decreasing NFBG as $\beta(L)$, which can easily be calculated from Eq. (2) as $\beta(z=L)=-4.56 \mathrm{ps}^{2} \mathrm{~cm}^{-1}$ for a given value of the initial chirp $\alpha_{0}=-0.01 \mathrm{THz}^{2}$. Having calculated the dispersion at the end point of the grating, the maximum compression factor, in the case of constant nonlinearity and no loss/gain, is determined by the ratio of the input to output dispersion. In this case, the compression factor turns out to be 7.24. The evolution of the Bragg soliton (9) with pulse compression is shown in Fig. 2. 


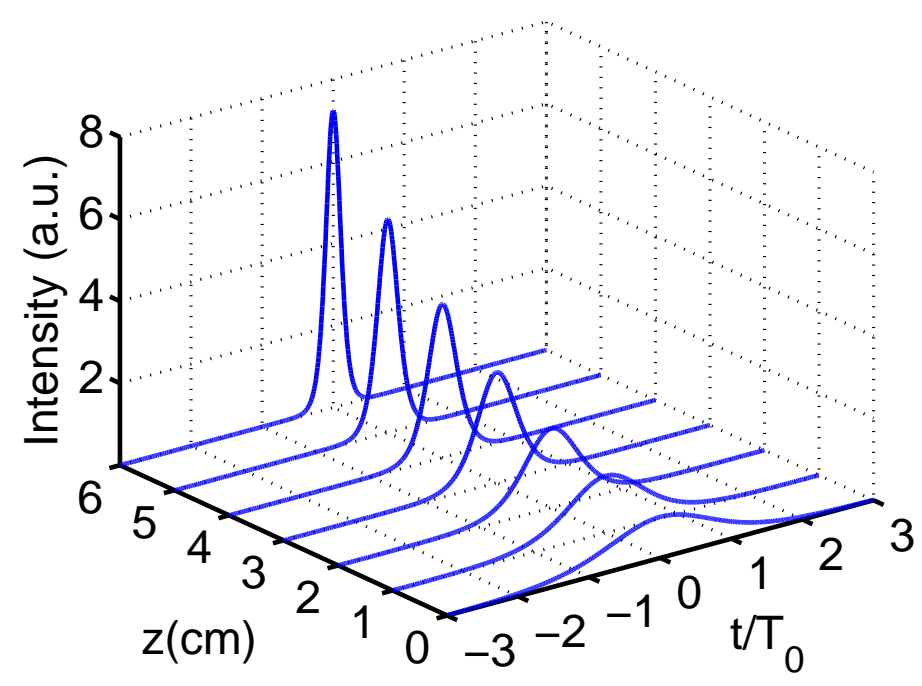

FIG. 2: 3D plot of bright Bragg soliton pulse compression for the physical parameters $\beta(0)=-33$ $\mathrm{ps}^{2} \mathrm{~cm}^{-1}, \alpha_{0}=-0.01 \mathrm{THz}^{2}, T_{0}=10 \mathrm{ps}$ and $z=6 \mathrm{~cm}$.

\section{CHIRPED HIGHER ORDER SOLITONS}

As is well known that the conventional solitons of NLSE with constant coefficients sustain both fundamental and higher order solitons, we envisage such fundamental and higher order soltions would exist for NLSE with dispersion varying profile too. Thus, in this section, using the chirped higher order solitons, we demonstrate a new type of pulse compression that exploits the combination of chirp and higher order soliton effect. We now proceed to generate the chirped higher order solitons by Bäcklund transformation method [24]. The chirped two-soliton in an exponentially decreasing dispersion medium is given by

$$
\psi(2)=\frac{N r_{1}-N r_{2}}{\mu D r} \exp \left(i \frac{\alpha_{0}}{2} t^{2}\right)
$$

where

$$
\begin{gathered}
N r_{1}=2 v_{1} \exp \left(i \theta_{1}\right) \operatorname{sech}\left(\zeta_{1}\right)\left[(\Delta u)^{2}+v_{1}^{2}-v_{2}^{2}+2 i(\Delta u) v_{2} \tanh \left(\zeta_{2}\right)\right], \\
N r_{2}=2 v_{2} \exp \left(i \theta_{2}\right) \operatorname{sech}\left(\zeta_{2}\right)\left[-(\Delta u)^{2}+v_{1}^{2}-v_{2}^{2}+2 i(\Delta u) v_{1} \tanh \left(\zeta_{1}\right)\right]
\end{gathered}
$$




$$
\begin{gathered}
D r=(\Delta u)^{2}+v_{1}^{2}+v_{2}^{2}-2 v_{1} v_{2} \tanh \left(\zeta_{1}\right) \tanh \left(\zeta_{2}\right)-2 v_{1} v_{2} \operatorname{sech}\left(\zeta_{1}\right) \operatorname{sech}\left(\zeta_{2}\right) \cos \left(\theta_{2}-\theta_{1}\right) \\
\zeta_{j}=\zeta_{j}(z, t)=-4 \beta_{0} \int u_{j}(z) v_{j}(z) d z+2 v_{j}(z) t \\
\theta_{j}=\theta_{j}(z, t)=2 \beta_{0} \int\left[u_{j}(z)^{2}-v_{j}(z)^{2}\right] d z-2 u_{j}(z) t,
\end{gathered}
$$

where

$$
\Delta u=u_{2}-u_{1} \quad \text { and } \quad j=1,2 .
$$

The parameters $\left(u_{1}, u_{2}\right)$ and $\left(v_{1}, v_{2}\right)$ are the physical parameters of the chirped two-soliton and they represent the velocities and amplitudes, respectively. As has been discussed in the chirped one-soliton, here also we assume the following values for the constants $\lambda_{0 I_{j}}=$ $1 /\left(2 T_{0 j}\right)$ and $\lambda_{0 R_{j}}=0$, where $j=1,2$. With these conditions, the chirped two-soliton becomes:

$$
\psi(2)=\sqrt{-\frac{\beta_{0}}{\gamma}} \frac{\exp \left(\alpha_{0} \beta_{0} z / 2\right)}{T_{01} T_{02}} \frac{F_{0}}{G_{0}}
$$

where

$$
\begin{gathered}
F_{0}=\left(T_{02}^{2}-T_{01}^{2}\right)\left[T_{02} e^{i m_{1}} \operatorname{sech}\left(\frac{t}{T_{01}} e^{\alpha_{0} \beta_{0} z}\right)-T_{01} e^{i m_{2}} \operatorname{sech}\left(\frac{t}{T_{02}} e^{\alpha_{0} \beta_{0} z}\right)\right], \\
G_{0}=T_{01}^{2}+T_{02}^{2}-T_{01} T_{02} \operatorname{sech}\left(\frac{t}{T_{01}} e^{\alpha_{0} \beta_{0} z}\right) \operatorname{sech}\left(\frac{t}{T_{02}} e^{\alpha_{0} \beta_{0} z}\right)\left[\cosh \left(\xi^{+}\right)-\cosh \left(\xi^{-}\right)+2 \cos (\delta \theta)\right],
\end{gathered}
$$

with

$$
m_{j}=\frac{1}{2}\left(\alpha_{0} t^{2}-\frac{1}{\alpha_{0} T_{0 j}^{2}}\right) e^{\alpha_{0} \beta_{0} z}, \quad \xi^{ \pm}=\frac{T_{02} \pm T_{01}}{T_{01} T_{02}} t e^{\alpha_{0} \beta_{0} z}, \quad \delta \theta=\frac{T_{02}^{2}-T_{01}^{2}}{2 \alpha_{0} T_{01}^{2} T_{02}^{2}} e^{\alpha_{0} \beta_{0} z} .
$$

It should be emphasized that the chirped solitons obtained by this method has already been correlated with the chirped solitons derived by the self-similar scaling analysis [10]. Based on this fact, the chirped two-soliton may emulate the self-similar property during the course of propagation along the exponentially decreasing dispersive media. This chirped two-soliton also enhances the chirp and maintains it during the propagation by establishing 
a balance between the exponentially growing chirp with that of the exponentially decreasing chirp induced by the exponentially decreasing dispersion. The highly enhanced linear chirp as well as the higher order soliton property can be utilized to achieve the pedestal-free compression which will be discussed in the following section.

\section{STUDIES ON CHIRPED HIGHER ORDER SOLITONS}

The theoretical formalism of these chirped higher order solitons will now be used to investigate pulse compression under the influence of chirping in the exponentially decreasing dispersive media. In the first sub-section (VIIA), we deal with the high degree of pulse compression by utilizing the general higher order soliton property as well as an initial chirp associated with the two-soliton. In the second sub-section (VIIB), we devote our attention on the compression by chirped higher order soliton breathers i.e., special hyperbolic secant pulses whose initial amplitude is twice that of a fundamental chirped soliton.

\section{A. Pulse Compression: Chirped Higher Order Solitons:-}

In this Section, we demonstrate pulse compression using higher order soliton as well as chirped soliton properties to achieve efficient compression. Further, we discuss pulse compression from the general chirped two-soliton of Eq. (12). The chirped higher order soliton pulses undergo either broadening or compression, depending on the sign of the initial frequency chirp parameter. Here, we analyze the compression of chirped two-soliton with the physical parameters, namely, initial chirp $\alpha_{0}$, dispersion $\beta_{0}$, velocities $u_{1}, u_{2}$ and amplitudes $v_{1}, v_{2}$. As the resulting chirped two-soliton develops relatively large chirp than the fundamental chirped soliton, highly compressed pulse, owing to higher order solitons, is possible after the combination of chirp compensation and higher order soliton effects.

As discussed in Section I, if $N$ is an integer larger than $1(N>1)$, the pulse evolution is very different from the case of a fundamental soliton $[2,3]$. Now, we discuss the physical mechanism of the periodic evolution of the two-soliton under the influence of chirp in the exponentially decreasing dispersive media. For all higher order solitons $(N>1)$, the effect of SPM dominates over the effect of AGVD. While the effect of SPM is to generate a positive chirp, the effect of AGVD is to produce a negative chirp. Due to the large intensity of 
the two-soliton, the amount of positive chirp generated by SPM is greater than that of the negative chirp produced by the AGVD. Indeed, the negative chirp due to AGVD decreases exponentially and at the same time, is maintained by the exponential growth of the external chirp. However, the resulting opposite chirps do not get cancelled out completely. As a result, the two-soliton pulses acquire a positive chirp. The impact of AGVD on a positively chirped pulse leads to the pulse compression [2].

Figure 3(a) shows the compression of chirped two-soliton for the following physical parameters: $\beta(0)=-20 \mathrm{ps}^{2} \mathrm{~km}^{-1}, \gamma=2 \mathrm{~W}^{-1} \mathrm{~km}^{-1}, \alpha_{0}=-0.01 \mathrm{THz}^{2}, u_{1}=0.05, u_{2}=0.025$, $v_{1}=0.025, v_{2}=0.15$ and $z=12 \mathrm{~km}$. Solid and dashed curves in Fig. 3(b) represent the initial and compressed pulses at $z=12 \mathrm{~km}$, respectively. In Fig. 3(c), the solid and dashed curves show the initial and compressed pulses at $z=12 \mathrm{~km}$ in logarithmic scale, respectively. The spectra of the initial (solid curve) and compressed (dashed curve) pulses are plotted in Fig. 3(d). Fig. 3(e) shows the behavior of compression factor (solid curve) and peak power (dashed curve) which grow approximately at the reciprocal of the decay rate of the dispersion (dot-dashed curve), i.e. the function $\exp \left(\alpha_{0} \beta_{0} z\right)$.

\section{B. Pulse Compression: Chirped Soliton Breathers}

In this subsection, we investigate pulse compression by a special pulse, which is also known as chirped soliton breather, whose initial amplitude is twice that of the fundamental soliton. The $N$-breather is a bound state of $N$-fundamental solitons. More generally, an $N$-soliton solution (higher order breather), with the pulse amplitudes $v_{j}=(2 j-1)$ for $j=1, \ldots, N$, has the shape $\psi=N \operatorname{sech}(t)$ when $z=0$ if the soliton phases are chosen properly. Note that there is no binding energy between the solitons in the breathers, i.e., the total energy of the breather is equal to the sum of the energies of its constituent solitons [2]. The continuous interaction of the $N$-solitons gives rise to the periodic behavior of the combined pulse. The most important and interesting special case occurs at $z=0$, when the eigenvalues are 1 and 3. Under these parametric conditions, the general two-soliton solution becomes a familiar hyperbolic secant shape whose initial amplitude is doubled i.e., $2 \operatorname{sech}(t)$. This special 2-soliton breather was first found by Satsuma and Yajima [25] and has also been observed in experiments in optical fibers and planar waveguides [26]. Similarly, we

also catch up with this type of special chirped 2-soliton breather pulse from the chirped 


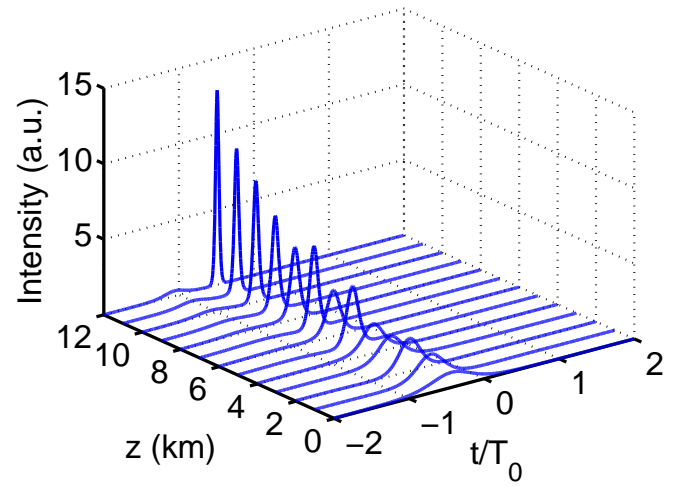

(a)

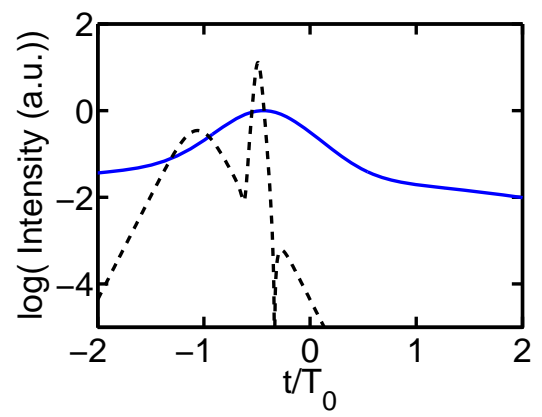

(c)

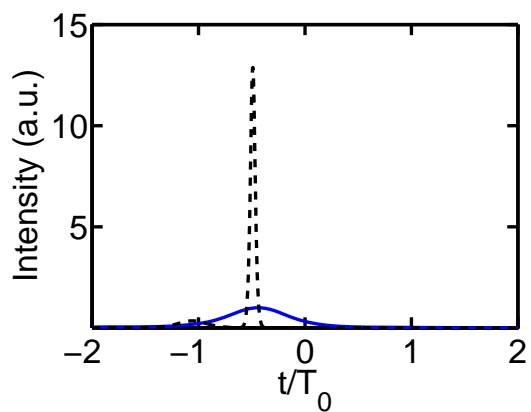

(b)

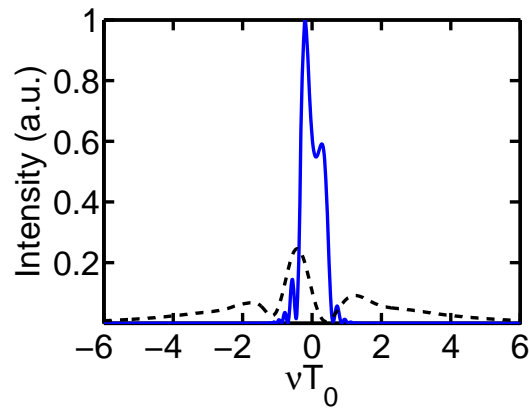

(d)

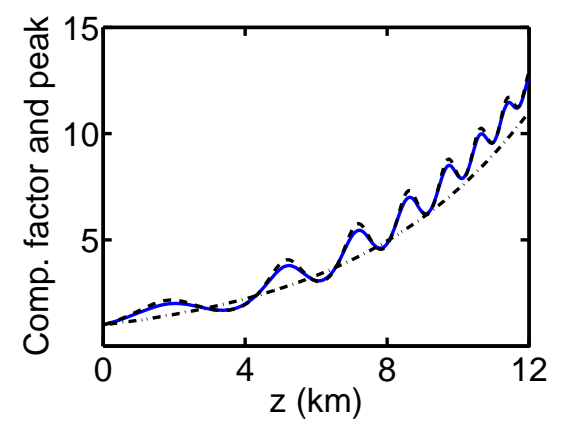

(e)

FIG. 3: (a) Compression of chirped two-soliton for the following physical parameters $\beta(0)=-20$ $\mathrm{ps}^{2} \mathrm{~km}^{-1}, \gamma=2 \mathrm{~W}^{-1} \mathrm{~km}^{-1}, \alpha_{0}=-0.01 \mathrm{THz}^{2}, u_{1}=0.05, u_{2}=0.025, v_{1}=0.025, v_{2}=0.15$ and $z=12 \mathrm{~km}$. (b) Solid and dashed curves represent initial and compressed pulses at $z=12 \mathrm{~km}$, respectively. (c) Solid and dashed curves represent initial and compressed pulses at $z=12 \mathrm{~km}$ in logarithmic scale, respectively. (d) Solid and dashed curves represent the spectra of initial and compressed pulses (e) The compression factor (solid curve) and peak power (dashed curve) follow the decay rate of the dispersion (dot-dashed curve): the function $\exp \left(\alpha_{0} \beta_{0} z\right)$. 
two-soliton solution at $z=0$. For the following choices of physical parameters (eigenvalues) $u_{1}=1 / 2 T_{0}, u_{2}=3 / 2 T_{0}$ and $v_{1}=v_{2}=0$, the general chirped two-soliton becomes a familiar hyperbolic secant shape whose amplitude is doubled that of a one-soliton,

$$
\psi(z=0, t)=\frac{2}{T_{0}} \sqrt{\frac{\beta_{0}}{\gamma}} \operatorname{sech}\left(\frac{t}{T_{0}}\right) \exp \left[i\left(\frac{\alpha_{0}}{2} t^{2}+\frac{\pi}{8}\right)\right],
$$

where $\alpha_{0}=-4 /\left(T_{0}^{2} \pi\right)$. The resulting chirped soliton breather differs from the conventional breather mainly because of the initial chirp associated with the special 'sech' type pulse. This is the simplest example of a higher order soliton breather $(N=2)$, where the initial amplitude is doubled compared to that of a fundamental soliton. This kind of initially chirped higher order soliton breather enjoys advantages over the fundamental soliton in optical communication systems, as it undergoes extremely high degree of pulse compression. We adopt the split-step Fourier method for studying the chirped 2-soliton breather and the simulation results do agree with the analytic solution very well. Fig. 4(a) shows the evolution of chirped 2-soliton breather in the exponentially decreasing dispersion for the following physical parameters: $T_{0}=10 \mathrm{ps}, \beta(0)=-20 \mathrm{ps}^{2} \mathrm{~km}^{-1}, \gamma=2 \mathrm{~W}^{-1} \mathrm{~km}^{-1}, u_{1}=1 / 2 T_{0}$, $u_{2}=3 / 2 T_{0}$ and $v_{1}=v_{2}=0$, and $z=12 \mathrm{~km}$. In Fig. 4(b), the solid and dashed curves represent the initial and compressed pulses at $z=12 \mathrm{~km}$, respectively. Fig. 4(c) explains the initial (solid curve) and compressed (dashed curve) pulses at $z=12 \mathrm{~km}$ in logarithmic scale, respectively. In Fig. 4(d), solid and dashed curves represent the spectra of initial and compressed chirped 2-soliton breather pulses. Fig. 4(e) represents the variation in the compression factor (solid curve) and peak power (dashed curve) which follow the reciprocal of

the decay rate of the dispersion (dot-dashed curve): the function $\exp \left(\alpha_{0} \beta_{0} z\right)$. The period of the chirped 2-soliton breather is found to be $z_{0}=\ln [1+1 /(2 k+1)] /\left(\alpha_{0} \beta_{0}\right)$, where $k=1,2,3 \ldots$. The 2 -sech pattern is repeated over each section of length $z_{0}$, where $z_{0}$ decreases with the propagation length.

\section{CONCLUSION}

We have employed the Painlevé analysis for determining the conditions for soliton pulse propagation in dispersion decreasing fiber media. The analytical results suggest the possibility of two dispersion profiles, namely, constant and exponentially varying dispersion profiles. For the exponentially scaled dispersion, the fiber system equation has a connection 


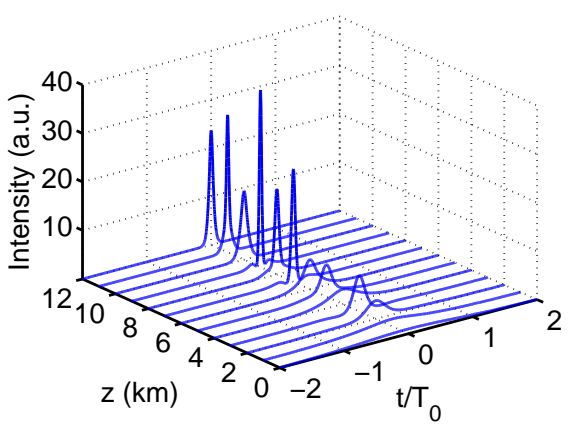

(a)

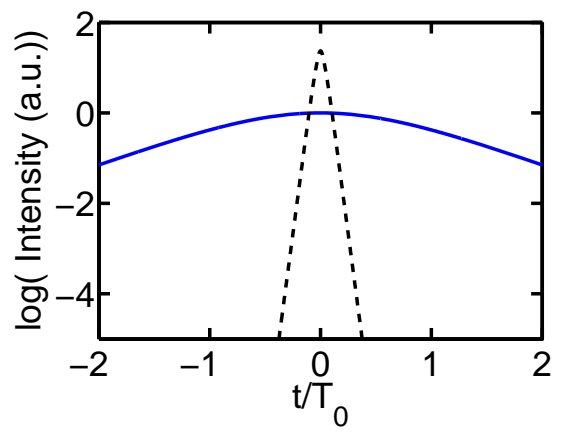

(c)

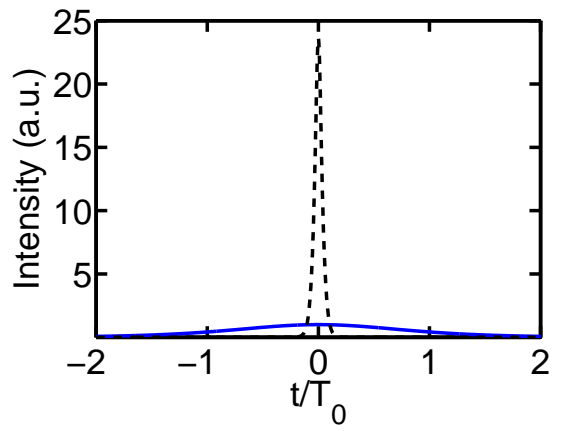

(b)

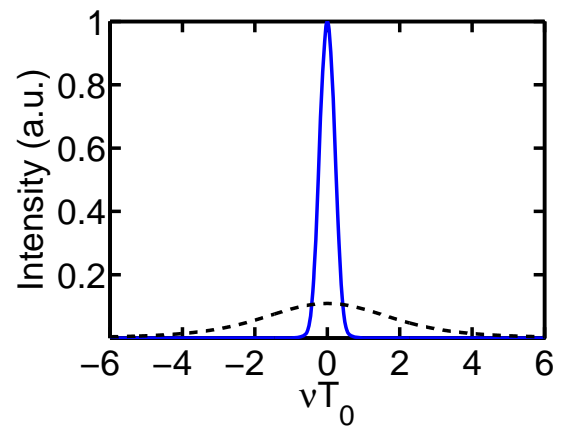

(d)

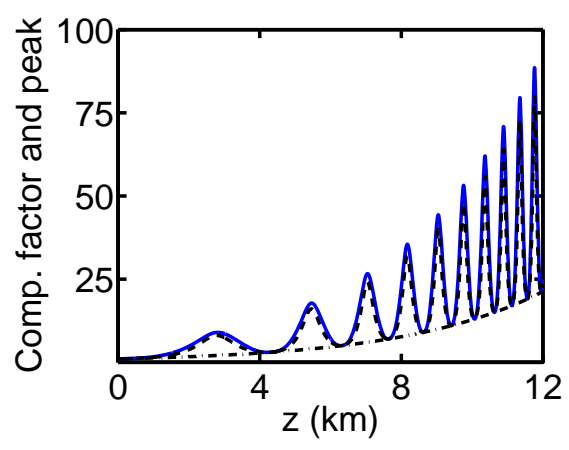

(e)

FIG. 4: (a) Compression of chirped 2-soliton breather for the following physical parameters $\beta(0)=$ $-20 \mathrm{ps}^{2} \mathrm{~km}^{-1}, \gamma=2 \mathrm{~W}^{-1} \mathrm{~km}^{-1}$ and $z=12 \mathrm{~km}$. (b) Solid and dashed curves represent initial and compressed pulses at $z=12 \mathrm{~km}$, respectively. (c) Solid and dashed curves represent initial and compressed pulses at $z=12 \mathrm{~km}$ in logarithmic scale, respectively. (d) Solid and dashed curves represent the spectra of initial and compressed pulses (e) The compression factor (solid curve) and peak power (dashed curve) follow the decay rate of the dispersion (dot-dashed curve): the function $\exp \left(\alpha_{0} \beta_{0} z\right)$. 
with the constant coefficient NLSE through another well-known variable coefficient NLSE. The explicit chirped one-soliton solution is generated by means of the Bäcklund transformation method. Furthermore, using the variational equation of the chirp, the formation of the chirped optical soliton has been discussed in detail. Further, we have presented an application of the chirped optical solitons for pulse compression through the dispersion decreasing nonlinear fiber Bragg grating. We believe that the results reported in this work could also be applicable to other physical systems governed by NLSE with varying dispersion profiles. In addition to the chirped fundamental soliton, we have also discussed the chirped two soliton solution for the exponentially decreasing dispersive media. Besides, we have investigated the compression process through the chirped soliton breathers. The authors are of the opinion that the investigations and results that have emanated of this work shall pave the way for realizing compact as well as efficient pulse compressor, capable of generating transform-limited pulses with incredibly negligible pedestals.

\section{Acknowledgements}

The support from The Hong Kong Polytechnic University (Project No. J-BB9M) is gratefully acknowledged. KN wishes to thank Prof. Miki Wadati and The Royal Society for the support in the form of International Short Visit funding.

[1] A. Hasegawa and F. Tappert, Appl. Phys. Lett. 23, 142 (1973).

[2] G. P. Agrawal, Nonlinear Fiber Optics (Academic Press, San Diego, 2001).

[3] L. F. Mollenauer and J. P. Gordon, Solitons in optical fibers: Fundamentals and Applications (Elsevier Academic Press, San Diego, 2006).

[4] V. E. Zakharov and A. B. Shabat, Sov. Phys.-JETP, 23, 142 (1972).

[5] D. J. Richardson, R. P. Chamberlin, L. Dong and D. N. Payne, Electron. Lett. 31, 1681 (1995).

[6] A. J. Stentz, R. W. Boyd and A. F. Evans, Opt. Lett. 20, 1770 (1995).

[7] G. P. Agrawal, Applications of Nonlinear Fiber Optics (Academic Press, San Diego, 2001).

[8] J. D. Moores, Opt. Lett. 21, 555 (1996). 
[9] V. I. Kruglov, A. C. Peacock and J. D.Harvey, Phys. Rev. E 71, 056619 (2005).

[10] Qian Li, K. Senthilnathan, K. Nakkeeran, and P. K. A. Wai, J. Opt. Soc. Am. B, 26, 432 (2009).

[11] Qian Li, P. K. A. Wai, K. Senthilnathan and K. Nakkeeran, J. Lightwave Tech, 29, 1293 (2011).

[12] L. Vázquez, L. Streit and V. M. Pérez-García, Eds., Nonlinear Schrödinger and Klein-Gordon systems: Theory and Applications, World Scientific, Singapore (1996).

[13] P. V. Mamyshev and S. V. Chernikov, Opt. Lett. 15, 1076 (1990).

[14] S. V. Chernikov, E. M. Dianov, D. J. Richardson, D. N. Payne, Opt. Lett. 18, 476 (1993).

[15] D. Anderson, M. Lisak, B. Malomed, and M. Quiroga-Teixeiro, J. Opt. Soc. Am. B 11, 2380 (1994).

[16] V. N. Serkin and A. Hasegawa, IEEE J. Sel. Top. Quantum Electron. 8, 418 (2002).

[17] P. A. Clarkson, Proc. Royal Soc. Edinburgh 109A, 109 (1988). M. J. Ablowitz and P. A. Clarkson, Solitons, Nonlinear Evolution Equations, and Inverse Scattering (Cambridge University Press, Cambridge, 1991). M. J. Ablowitz, D. J. Kaup, A. C. Newell and H. Segur, Phys. Rev. Lett., 30, 1262 (1973); Phys. Rev. Lett., 31, 125 (1973).

[18] S. P. Burtsev, V. E. Zakharov, and A. V. Mikhailov, Theor. Math. Phys. 70, 227 (1987).

[19] K. Nakkeeran, J. Phys. A: Mathematical and General 34, 5111 (2001).

[20] C. C. Mak, K. W. Chow and K. Nakkeeran, J. Phys. Soc. Jpn. 74, 1449 (2005).

[21] B. A. Malomed, Progress in Optics, 43, 71 (2003).

[22] E. N. Tsoy and C. M. de Sterke, Phys. Rev. E 62, 2882 (2000).

[23] G. Lenz and B. J. Eggleton, J. Opt. Soc. Am. B 15, 2979 (1998).

[24] A. A. Rangwala and J. A. Rao, Phys. Lett. A, 112, 188 (1985).

[25] J. Satsuma and N. Yajima, Prog. Theor. Phys. (Suppl.) 55, 62 (1974).

[26] N. N. Akhmediev and A. Ankiewicz, Solitons: Nonlinear pulses and beams (Chapman and Hall, London, 1997). 